\title{
Características clínicas, epidemiológicas y patológicas de los linfomas en pacientes del Instituto Nacional de Salud del Niño de Breña - Perú,
}

\section{5-2019 \\ Clinical, epidemiological and pathological characteristics of lymphomas in patients from the National Institute of Child Health of Breña - Peru, 2015-2019}

\author{
Cristina Noelia Gálvez Paz ${ }^{1, a}$, Mariela Esther Mendoza Goycochea ${ }^{1, b}$, Nora Espíritu, ${ }^{1,2,}$, Edith Maritza Paz Carrillo, 3,,d \\ ${ }^{1}$ Facultad de Medicina Humana, Universidad San Martin de Porres. Lima, Perú. \\ ${ }^{2}$ Hospital Nacional Dos de Mayo. Lima, Perú. \\ ${ }^{3}$ Instituto Nacional de Salud del Niño de Breña. Lima, Perú. \\ ${ }^{4}$ Facultad de Medicina, Universidad Nacional Mayor de San Marcos. Lima, Perú. \\ ${ }^{a}$ Estudiante de medicina, ORCID: https://orcid.org/0000-0002-1130-9826 \\ ${ }^{\mathrm{b}}$ Estudiante de medicina, ORCID: https://orcid.org/0000-0003-3695-8387 \\ 'Médica pediatra, magister en salud pública. ORCID: https://orcid.org/0000-0002-6340-0285 \\ ${ }^{\text {d} M e ́ d i c a ~ a n a t o m o ~ p a t o ́ l o g a, ~ m a g i s t e r ~ e n ~ s a l u d ~ p u ́ b l i c a ~ y ~ g e s t i o ́ n ~ s a n i t a r i a . ~ O R C I D: ~ h t t p s: / / o r c i d . o r g / 0000-0003-2740-0867 ~}$
}

An Fac med. 2021;82(1):27-33. / DOI: https://doi.org/10.15381/anales.v82i1.19362

\section{Correspondencia:}

Cristina Noelia Gálvez Paz cristinagpaz55@gmail.com

Recibido: 29 de diciembre 2020 Aprobado: 15 de mayo 2021 Publicación en línea: 10 de junio 2021

Conflictos de interés: Los autores declaran no tener conflictos de interés.

Fuente de financiamiento: Autofinanciado

El presente artículo forma parte de la tesis que será presentada por Cristina Noelia Gálvez Paz y Mariela Esther Mendoza Goycochea, para optar el Título Profesional de Médico Cirujano de la Facultad de Medicina de la Universidad San Martín de Porres.

Citar como: Gálvez C, Mendoza M, Espiritu N, Paz E. Características clínicas, epidemiológicas y patológicas de los linfomas en pacientes del Instituto Nacional de Salud del Niño de Breña - Perú, 2015-2019. An Fac med. 2021;82(1):27-33. DOI: https://doi. org/10.15381/anales.v82i1.19362
Resumen

Objetivo. Determinar las características clínicas, epidemiológicas y patológicas de los linfomas en pacientes del Instituto Nacional de Salud del Niño Breña entre el 2015-2019. Métodos. Se realizó un estudio cuantitativo, observacional, transversal, descriptivo y retrospectivo; correspondiente a los pacientes pediátricos con diagnóstico de linfoma entre los años 2015 al 2019. Para el análisis se utilizó el programa SPSS v24. Las variables cuantitativas fueron expresadas en medidas de tendencia central y las cualitativas en frecuencias absolutas y relativas. Resultados. Se incluyeron 43 casos, el promedio fue 7,2 años (1a - 16a), el 72,1\% $(n=31)$ fueron del sexo masculino. Entre los sintomas más frecuentes están fiebre $51,2 \%(n=22)$, baja de peso $41,9 \%(n=18)$ y cansancio $20,9 \%(n=9)$ y entre los signos prevalentes, las linfadenopatías $83,7 \%(n=$ 36) y tumoración abdominal con $14 \%(n=6)$. La localización más frecuente fue la de tipo ganglionar con $69,8 \%(n=30)$. La anemia se presentó en un $58,1 \%(n=25)$. Según el diagnóstico anátomopatologico, 20 $(48,8 \%)$ casos fueron Linfoma Hodgkin (LH) y $23(51,2 \%)$ no Hodgkin (LNH); siendo los tipos más frecuente el linfoma linfoblástico B con un 25,6\% ( $n=11)$, seguido de LH celularidad mixta con $20,9 \%(n=9)$. El linfoma más frecuente en etapa preescolar fue el LH celularidad mixta con $31,3 \%(n=5)$, mientras que en etapa escolar el linfoma linfoblástico B con 25,9\% (n=7). Conclusiones. En pacientes pediátricos, el linfoma afectó mayormente al sexo masculino, presentando fiebre, baja de peso, linfoadenopatías, tumoración abdominal y anemia. El linfoma linfoblástico B fue el más frecuente; estas características clínicas son similares a lo reportado a nivel mundial.

Palabras clave: Linfoma; Linfoma no Hodgkin; Epidemiología Descriptiva; Pediatría; Morbilidad; Anemia; Fiebre; Linfadenopatía; Perú (fuente: DeCS BIREME).

\section{Abstract}

Objective. To determine the clinical, epidemiological and pathological characteristics of lymphomas in patients of the National Institute of Child Health Breña between 2015-2019. Methods. A quantitative, observational, crosssectional, descriptive and retrospective study was carried out; corresponding to pediatric patients diagnosed with lymphoma between 2015 and 2019. The SPSS v24 program was used for the analysis. The quantitative variables were expressed in measures of central tendency and the qualitative ones in absolute and relative frequencies. Results. 43 cases were included, the average age was 7.2 years $(1 \mathrm{a}-16 \mathrm{a}), 72.1 \%(n=31)$ were male. Among the most frequent symptoms are fever $51.2 \%(n=22)$, weight loss $41.9 \%(n=18)$ and fatigue $20.9 \%$ $(n=9)$ and among the prevalent signs, lymphadenopathy $83.7 \%(n=36)$ and abdominal mass with $14 \%(n=6)$. The most frequent location was the lymph node type with $69.8 \%(n=30)$. Anemia occurred in $58.1 \%(n=25)$. According to the pathological diagnosis, $20(48.8 \%)$ cases were Hodgkin's Lymphoma (HL) and $23(51.2 \%)$ were non-Hodgkin's (NHL); the most frequent types being B lymphoblastic lymphoma with $25.6 \%(n=11)$, followed by mixed cellularity $\mathrm{LH}$ with $20.9 \%(n=9)$. The most frequent lymphoma in preschool stage was mixed cellularity $\mathrm{LH}$ with $31.3 \%(n=5)$, while in school stage B lymphoblastic lymphoma with $25.9 \%(n=7)$. Conclusions. In pediatric patients, lymphoma mainly affects the male sex, presenting fever, weight loss, lymphadenopathy, abdominal tumor and anemia. B lymphoblastic lymphoma was the most frequent; these clinical characteristics are similar to those reported worldwide.

Keywords: Lymphoma; Non-Hodgkin Lymphoma; Epidemiology, Descriptive; Pediatrics; Morbidity; Anemia; Fever; Lymphadenopathy; Peru (source: MeSH NLM). 


\section{INTRODUCCIÓN}

Los linfomas se manifiestan como producto de una proliferación maligna de las células linfoides. Se trata de una agrupación diversa de neoplasias, con una gama extensa de estructuras, lo que causa una variedad de presentaciones clínicas, que parten desde aspectos casi leucémicos -con daño extenso del organismo-, a otros netamente circunscritos. En contraste con las leucemias, el desarrollo maligno se origina desde que la célula hematopoyética deja la médula ósea ${ }^{(1)}$.

A nivel mundial, los linfomas constituyen la tercera neoplasia maligna infantil más común, precedida de las leucemias y los tumores cerebrales. Su incidencia se incrementa con la edad y tiene mayor predisposición en varones con una relación 2:1 respecto a las niñas ${ }^{(1)}$. Estadísticas del Instituto Nacional de Cáncer en los Estados Unidos estiman que hay cerca de 20 casos de LNH por cada 100000 personas dentro de la población estadounidense. Por su parte, el LH es relativamente raro, con alrededor de tres casos por cada 100000 personas. Es decir que aproximadamente $90 \%$ de los linfomas son del tipo LNH mientras que un $10 \%$ son del $\mathrm{LH}^{(2)}$.

Según el Registro de Cáncer de Lima Metropolitana, en el periodo 2010 2012, la incidencia de cáncer en niños fue de 162,9 por millón y 142 por millón para las niñas. Más de un tercio (40,2\%) de todas las neoplasias malignas fueron leucemias, con una incidencia de 62,2 por millón, seguido por tumores de sistema nervioso central $(20,4 \%)$ y linfomas $(11,1 \%)$. Respecto a los linfomas, estuvieron asociados a la infección por el virus de Epstein Barr, patología de alta incidencia en el país, similar a lo que ocurre en países asiáticos ${ }^{(3)}$.

En el Noreste de Nigeria, Yakubu M, en 2015, realizó un análisis retrospectivo de casos de linfomas en la infancia durante un período de 15 años, obtuvo 50 casos de linfoma, 10 (20\%) pertenecieron a linfoma de Hodgkin (LH) y 40 (80\%) a linfoma no Hodgkin (LNH). Demostró que el linfoma es común en hombres. El hueso maxilar fue el sitio más frecuente de malignidad primaria (36\%) y se observó una presentación tardía de los pacientes. Evidenció una nueva tendencia, en los pacientes con LNH a presentar con mayor frecuencia síntomas graves que en los que fueron diagnosticados con $\mathrm{LH}$. La mayoría con LH, eran de tipo predominante linfocítico, mientras que, en el LNH, predominaba el linfoma de células pequeñas no hendidas (Burkitt) con un70\%. Se concluyó que el linfoma infantil tuvo un ligero cambio en la presentación clínica, especialmente del LNH; los pacientes en este estudio tuvieron presentación tardía (4).

En Colombia, Tovar y Gómez, en 2018, realizaron un estudio descriptivo sobre la incidencia de cáncer infantil. Hallaron un total de 350 neoplasias en niños, teniendo en cuenta la Clasificación Internacional de Cáncer Infantil. El 37\% pertenecieron a casos de leucemia (Grupo I); 23\%, agruparon tumores sólidos (Grupo III); y el 17\%, abarcaron linfomas (Grupo II). En el Grupo II, conformados por los linfomas, de 47 casos, el $68 \%$ lo o conformaban hombres y el $51 \%$ pacientes de 10 a 14 años; la tasa de incidencia fue de 74 casos por cada millón de habitantes, reflejando una media de 15 casos por miIlón anualmente. En el grupo II, el linfoma de Burkitt presentó un 28\% del total de linfomas; sin embargo, en el total neoplasias, alcanzó un 4\%. Este tipo fue el menos frecuente abarcando un 3,7\% de cáncer en niños. En un 13\% existieron casos de neoplasias en niños y su incidencia fue de 15 afectados por millón, similar a un estudio realizado en los EE.UU. con el valor de 15,2 por millón ${ }^{(5)}$.

En Japón, Worawut y col., en 2019, realizaron un estudio observacional, descriptivo y transversal. Estudiaron a niños menores de 15 años diagnosticados con linfoma, entre enero de 1998 al 2014, los cuales seguían con tratamiento hasta el 31 de enero de 2016. El estudio se basó en las características iniciales y de largo plazo; 121 niños fueron diagnosticados con linfoma, de los cuales 114 llegaron ser seleccionados para el análisis. Concluyeron que la mayor parte eran varones, con una relación de 2:1; además se halló que en linfoma de Burkitt era el tipo más común de los LNH en pre adolescentes, mientras que, el linfoma difuso de células B y el linfoma de células grandes se presentaron en mayor porcentaje en la adolescencia. El diagnóstico se basó en la histopatología, características inmunológicas y genética molecular ${ }^{(6)}$.

En Perú, Padilla y col., realizaron un estudio descriptivo, retrospectivo, tipo serie de casos de 433 pacientes portadores de linfoma del año 1998 al 2008, con el objetivo de conocer las características clínicas, epidemiológicas y patológicas de los pacientes diagnosticados con linfoma en el Hospital Nacional Cayetano Heredia. El estudio tuvo como resultados relevantes el promedio de edad, el cual fue de 44,8 años, donde el 60,04\% pertenecía al sexo masculino; el lugar de nacimiento y procedencia fue Lima con $61,5 \%$ y $90 \%$ respectivamente. Los síntomas más característicos fueron la presencia de linfoadenopatías $(3,7 \%)$ y baja de peso $(40,5 \%)$. Los pacientes con estadio clínico I - II fueron un 55,08\%. La localización ganglionar fue la más frecuente, con un 55,1\%. Las localizaciones con mayor repetición fueron la gastrointestinal con un $15,2 \%$ y la de piel con un $10,8 \%$. De los LNH, el inmunofenotipo B tuvo un $57,8 \%$ de frecuencia y el patrón histológico más característico fue el linfoma de células grandes difuso con 35,8\%. La enfermedad de Hodgkin tuvo como porcentaje solo un $14,08 \%$. De los pacientes tratados, el 78,12\% tuvo una respuesta completa y parcial. El tratamiento con adriamicina, bleomicina, vincristina y dacarbazina (ABVD) en la enfermedad de Hodgkin tuvo como respuesta completa y parcial un 90,97\%. La conclusión fue que los pacientes con linfoma atendidos en el Hospital Nacional Cayetano Heredia tuvieron las mismas características a lo reportado a nivel mundial ${ }^{(7)}$.

El linfoma sigue teniendo una incidencia importante en adultos, como lo reportado en el Hospital Cayetano Heredia; sin embargo, en nuestro país no se cuenta con estudios en edad pediátrica. Es por ello que el presente artículo tiene como objetivo determinar las características clínicas, epidemiológicas y patológicas de los pacientes con diagnóstico de linfoma atendidos en el Instituto Nacional de Salud del Niño de Breña (INSN) entre los años 2015-2019 en Lima, Perú. 


\section{MÉTODOS}

Se realizó un estudio cuantitativo, observacional, transversal, descriptivo y retrospectivo. La población de estudio fueron los pacientes con diagnóstico de linfoma en el INSN entre los años 20152019, según el registro de la Oficina de Estadística e Informática y del registro del Servicio de Anatomía Patológica del INSN Breña.

\section{Población y muestra del estudio}

Durante dicho periodo de estudio se registraron 54 casos de linfoma, de los cuales 43 cumplieron con los criterios de selección. Los criterios de inclusión fueron:

- Pacientes con diagnóstico anátomo patológico de linfoma entre 2015 al 2019, según el servicio de anatomía patológica

- Pacientes entre 0 a 18 años

- Datos completos de la historia clínica del paciente

Mientras que los criterios de exclusión fueron:

- Niños con recidiva de linfoma y tratamiento anteriores.

- Niños que presentaron asociado otro tumor maligno

\section{Técnicas y procedimientos de recolección de datos}

Se empleó una ficha de recolección de datos como instrumento, para recolectar las siguientes variables: edad, sexo, procedencia, antecedentes, signos y síntomas al ingreso, hemograma, localización del tumor y diagnóstico anatomopatológico. Cabe precisar que el INSN de Breña cuenta con estudios de patología completa para subclasificar los linfomas, las muestras son enviadas al Instituto Nacional de Enfermedades Neoplásicas (INEN) para verificación del diagnóstico y tratamiento correspondiente.

\section{Análisis estadístico}

Los datos obtenidos a partir de las historias clínicas y fichas de recolección fueron tabulados utilizando el programa
Excel y analizados con el programa SPSS versión 24. Se realizó un análisis univariado, las variables cualitativas se expresaron en frecuencias y porcentajes y las cuantitativas en medidas de tendencia central.

\section{Consideraciones éticas}

La investigación fue aprobada por el Comité de Ética de la Facultad de Medicina Humana de la Universidad San Martin de Porres y el Comité de Ética en Investigación del Instituto Nacional de Salud del Niño de Breña. El estudio estuvo sujeto a las normas de buena práctica clínica y cumplió con las normas éticas. Las identidades de los pacientes se codificaron para conservar el anonimato. Además, no implicó ningún tipo de riesgo para los pacientes dado que se utilizaron solo sus historias clínicas.

\section{RESULTADOS}

Se obtuvo información de 43 pacientes, de los cuales el 72,1\% ( $n=31)$ fueron del sexo masculino y el $27,9 \%(n=12)$ del sexo femenino. El promedio de edad fue 7,2 años (1 año - 16 años). El 62,8\% $(n=27)$ fueron escolares y los restantes pre escolares. El 32,6\% ( $n=14)$ procedían de Lima, seguidos de la costa $(30,2 \%)$ excluyendo Lima; luego la selva con igual porcentaje que la sierra $(18,6 \%)$ (Tabla 1$)$.
Los síntomas principales fueron fiebre y baja de peso con $51,2 \%$ ( $n=22)$ y $41,9 \%$ $(n=18)$ respectivamente, seguido de cansancio $20,9 \%(n=9)$, tos y dolor abdominal con $18,6 \%(n=8)$ cada uno. Mientras que las linfadenopatías fueron el signo más frecuente con un $83,7 \%(n=36)$ seguido de tumoración abdominal 14\% $(n=9)$. En las características clínicas, en antecedentes, la opción ninguna destacó con un $71,1 \%$. La localización que predominó fue la ganglionar con $69,8 \%(n=30)$; y en laboratorio, la anemia prevaleció con un 58,1\% (n=25) (Tabla 2).

En el diagnostico anatomopatológico, los LH fueron 20 casos (48,8\%), representados por el LH celularidad mixta, LH esclerosis nodular y $\mathrm{LH}$ rico en linfocitos. Los LNH fueron 23 casos (51,2\%), representados por el linfoma linfoblastico " $T$ ", linfoma linfoblastico "B", linfoma de Burkitt, linfoma difuso de células grandes y linfoma anaplasico de células grandes (Tabla 3).

En relación a los $\mathrm{LH}$, los más frecuentes fueron los LH de celularidad mixta con 9 casos ( $n=20,9 \%$ ) y los LH esclerosis nodular con 7 casos ( $n=16,3 \%$ ). Para los LNH fue el linfoma linfoblástico B con 11 casos $(25,6 \%)$, seguido del linfoma linfoblastico " $T$ " con 5 casos $(11,6 \%)$ (Tabla 4).

Según grupos etarios, en pre escolares hubo 16 casos, $9 \mathrm{LH}$ y $7 \mathrm{LNH}$, siendo el más frecuente en los LH el de celularidad

Tabla 1. Características epidemiológicas de niños con diagnóstico de linfoma atendidos en el Instituto Nacional de Salud del Niño de Breña, 2015 - 2019. Lima, Perú

\begin{tabular}{lll} 
Características epidemiológicas & $\begin{array}{c}\text { Número de casos } \\
(\mathbf{N = 4 3 )}\end{array}$ & $\begin{array}{c}\text { Porcentaje } \\
\text { (\%) }\end{array}$ \\
\hline Edad & 27 & $(62,8)$ \\
\hline Escolar & 16 & $(37,2)$ \\
\hline Pre-escolar & & $(72,1)$ \\
\hline Género & 31 & $(27,9)$ \\
\hline Masculino & 12 & $(32,6)$ \\
\hline Femenino & & $(30,2)$ \\
\hline Procedencia & 14 & $(18,6)$ \\
\hline Lima & 13 & $(18,6)$ \\
\hline Sierra & 8 & \\
\hline
\end{tabular}

*Excepto Lima 
Tabla 2. Características clínicas de niños con diagnóstico de linfoma atendidos en el Instituto Nacional de Salud del Niño de Breña, 2015 - 2019. Lima, Perú.

\begin{tabular}{|c|c|c|}
\hline Características clínicas & $\begin{array}{l}\text { Número de casos } \\
\qquad(\mathrm{N}=43) \\
n\end{array}$ & $\begin{array}{c}\text { Porcentaje } \\
\text { (\%) }\end{array}$ \\
\hline \multicolumn{3}{|l|}{ Síntomas } \\
\hline Fiebre & 22 & $(51,2 \%)$ \\
\hline Baja de peso & 18 & $(41,9)$ \\
\hline Cansancio & 9 & $(20,9)$ \\
\hline Tos & 8 & $(18,6)$ \\
\hline Dolor abdominal & 8 & $(18,6)$ \\
\hline Diarrea & 7 & $(16,3)$ \\
\hline Disnea & 6 & $(14)$ \\
\hline Disfagia & 4 & $(9,3)$ \\
\hline Sudoración Nocturna & 3 & $(7)$ \\
\hline \multicolumn{3}{|l|}{ Signos } \\
\hline Linfadenopatias & 36 & $(83,7)$ \\
\hline Tumoración toraco abdominal & 6 & $(14)$ \\
\hline Hepatomegalia & 3 & $(7)$ \\
\hline \multicolumn{3}{|l|}{ Antecedentes } \\
\hline Ninguno & 31 & $(71,1)$ \\
\hline Resfrios & 7 & $(16,3)$ \\
\hline Tuberculosis & 3 & $(7)$ \\
\hline Varicela & 1 & $(2,3)$ \\
\hline Citomegalovirus & 1 & $(2,3)$ \\
\hline \multicolumn{3}{|l|}{ Localización } \\
\hline Ganglionar & 30 & $(69,8)$ \\
\hline Extraganglionar & 10 & $(23,3)$ \\
\hline Ambas & 3 & $(7)$ \\
\hline \multicolumn{3}{|l|}{ Laboratorio } \\
\hline Anemia & 25 & $(58,1)$ \\
\hline Ninguna & 10 & $(23,3)$ \\
\hline Anemia, Leucopenia y Plaquetopenia & 8 & $(18,6)$ \\
\hline
\end{tabular}

mixta con 5 casos (31,3\%) y en los LNH el linfoma linfoblástico B con 4 casos (25\%). En etapa escolar, los casos de linfomas fueron más numerosos, 27 casos de los cuales 11 fueron $\mathrm{LH}$ y $16 \mathrm{LNH}$; siendo en los LH, el más frecuente el LH de celularidad mixta y el $\mathrm{LH}$ de esclerosis nodular con 4 casos $(14,8 \%)$ cada uno; para el LNH al igual que en los pre escolares también fue frecuente con 7 casos $(25,9 \%)$ el linfoma linfoblástico B (Tabla 5).

Los linfomas más frecuentes en el sexo masculino fueron el $\mathrm{LH}$ celularidad mixta con un $29 \%(n=9)$, seguido de linfoma linfoblástico B con un $25,8 \%(n=8)$; mientras que en el sexo femenino el más frecuente fue linfoma linfoblástico $T$ y linfoma linfoblástico B, con un 25\% ( $n=3)$ cada uno (Tabla 6).

\section{DISCUSIÓN}

Los linfomas constituyen la tercera neoplasia maligna infantil más común después de la leucemia y tumor cerebral. Su incidencia aumenta con la edad y es más frecuente en varones con una relación $2: 1$ respecto a las niñas ${ }^{(1)}$, lo que se evidenció en esta investigación, siendo los casos de linfoma en el sexo masculino el de Salud del Niño de Breña, 2015-2019. Lima, Perú.
$72,1 \%$, similar a lo reportado por Yabuku, Tovar y Worawut ${ }^{(4,5,6)}$. La edad con mayor prevalencia que se encontró en nuestro estudio fueron los escolares (6 a 17 años) con un $62,8 \%$, similar a lo encontrado por Tovar en su estudio en el que la población infantil entre 10 a 14 años presentó mayor incidencia diagnóstica ${ }^{(5)}$.

Con respecto a las características clínicas, se hallaron como síntomas principales la fiebre y baja de peso con $51,2 \%$ y $41,9 \%$ respectivamente, mientras que la linfadenopatía fue el signo más frecuente con un $83,7 \%$, de los cuales un $69,8 \%$ fueron de localización ganglionar. Según lo reportado por Del Castillo, las ubicaciones con mayor frecuencia se encontraban en los ganglios con un 65,9\%, también se presentaron localizaciones fuera de los ganglios, dándose mayor aparición en piel y zona de la nariz ${ }^{\left({ }^{8}\right)}$. Según Padilla y col., en los síntomas más comunes destacaron la baja de peso y la presencia de linfadenopatías, similar a lo que se describe en otros estudios como el de Mozaffer; sin embargo, en estos estudios también se observó que síntomas como fiebre, anemia, hepatomegalia y esplenomegalia tuvieron una alta importancia en el diagnóstico ${ }^{(7)}$.

Con respecto a la localización, en nuestro estudio se observó una leve predominancia de los linfomas ganglionares sobre los extras ganglionares, estos últimos representaron el 23,3\%. Dentro de las localizaciones extra ganglionares se conserva la misma relación que las presentadas por Chang, quien encontró que la localización gastrointestinal y de piel fueron las más frecuentes con 38,6\% y $15,5 \%$ respectivamente ${ }^{(9)}$. Según el estudio de Boris Bencomo, la localización abdominal fue la más frecuente seguida por las localizaciones mediastinal y ganglionar ${ }^{(10)}$. En nuestro estudio 6 pacientes presentaron tumoración abdominal.

Tabla 3. Diagnóstico anatomopatológico de linfomas en niños atendidos en el Instituto Nacional

\begin{tabular}{lcc} 
Diagnóstico anatomopatológico & $\begin{array}{c}\text { Número de casos } \\
(\mathbf{n}=\mathbf{4 3 )}\end{array}$ & $\begin{array}{c}\text { Porcentaje } \\
(\mathbf{\%})\end{array}$ \\
\hline Linfoma Hodgkin & 20 & $(48,8)$ \\
\hline Linfoma no Hodgkin & 23 & $(51,2)$ \\
\hline Total & 43 & $(100)$ \\
\hline
\end{tabular}


Tabla 4. Diagnóstico anatomopatológico de linfomas en los niños atendidos en el Instituto Nacional de Salud del Niño de Breña, 2015- 2019. Lima, Perú.

\begin{tabular}{|c|c|c|}
\hline $\begin{array}{l}\text { Diagnóstico } \\
\text { anatomopatologico }\end{array}$ & $\begin{array}{l}\text { Número de casos } \\
(n=43)\end{array}$ & $\begin{array}{c}\text { Porcentaje } \\
(\%)\end{array}$ \\
\hline \multicolumn{3}{|l|}{ Linfoma Hodgkin } \\
\hline LH Celularidad mixta & 9 & $(20,9)$ \\
\hline LH Esclerosis Nodular & 7 & $(16,3)$ \\
\hline LH Rico en linfocitos & 4 & $(11,6)$ \\
\hline \multicolumn{3}{|l|}{ Linfoma No Hodgkin } \\
\hline Linfoma Linfoblastico B & 11 & $(25,6)$ \\
\hline Linfoma Linfoblastico T & 5 & $(11,6)$ \\
\hline Linfoma de Burkitt & 3 & (7) \\
\hline Linfoma Anaplasico de celulas grandes & 3 & (7) \\
\hline Linfoma difuso de células grandes & 1 & $(2,3)$ \\
\hline
\end{tabular}

Según la Sociedad Americana de Cáncer, la frecuencia del linfoma de Burkitt endémico y no endémico es $50 \%$; linfoma linfoblástico 20\%; linfoma de células grandes tipo B 10\%; y linfoma de células grandes anaplásicas $10 \%{ }^{(11)}$. Worwut y col. en su estudio realizado en Japón en 114 niños con linfoma, el 20\% fueron LH y el $80 \% \mathrm{LNH}^{(6)}$; en nuestro estudio, el $48,8 \%$ fueron $\mathrm{LH}$ y el $51,2 \% \mathrm{LNH}$, siendo los tipos más frecuentes linfoma linfoblástico B con 11 casos (25,6\%), LH celularidad mixta con 9 casos $(20,9 \%)$ y LH esclerosis nodular 7 casos (16,3\%). Tanto Yabuku como Tovar y Worawut, cuyos estudios fueron en población pediátrica, encontraron que de los LNH el más frecuente fue el de Burkitt; en nuestro estudio encontramos que fue el L. linfoblástico B. Padilla y col., cuyo trabajo fue en población mayormente adulta en el Perú, de los 433 pacientes estudiados, 85,92\% tuvieron LNH y $14,08 \% \mathrm{LH}$; de los LNH el $57,8 \%$ correspondía a inmunofenotipo $B$; $19,6 \%$, inmunofenotipo $T$ y un grupo de linfomas indeterminados $22,6 \%$. Según el tipo histológico, el más frecuente fue el LNH difuso $(35,79 \%)$. La literatura menciona que el linfoma linfoblástico se presenta con mayor frecuencia en hombres jóvenes, siendo una forma rara y agresiva de LNH. Actualmente, la OMS presenta una clasificación en donde el linfoma linfoblástico se agrupa junto a la leucemia linfoblástica aguda, pero difiere de esta por presentar menos de $20 \%$ de blastos medulares. Siendo la gran parte de casos de linfoma linfoblástico, las células $T$ con un $80 \%$; el resto son de linaje $\mathrm{B}^{(12)}$. El linfoma de Burkitt se caracteriza por presentar ensanchamiento mediastinal, sin calcificaciones, bordes policíclicos, derrame pleural y pericárdico con adenopatías cervicales, axilares y supraclaviculares, constituyendo una urgencia oncológica por compresión de estructura mediastinales ${ }^{(13)}$

Según el Instituto Nacional de Cáncer de los EE.UU., de los LH, el de esclerosis nodular, se presenta con mayor frecuencia en niños mayores y en adolescentes; en nuestro estudio, de 7 casos de este tipo de linfoma, 4 se presentaron en escolares. El LH con celularidad mixta, indica el instituto, que es frecuente en los niños menores de 10 años; nosotros encontramos que, de los 9 casos, 5 fueron en pre escolares y el resto en escolares ${ }^{(14)}$.

Según un grupo colaborativo español existe una relación de la clase social con la incidencia del LH; así, refieren que el LH de celularidad mixta es más frecuente en países económicamente más deprimidos, sobre todo cuando afecta a edades más tempranas, como es el caso de nuestro estudio, el cual predominó con 9 casos. Por otra parte, el subtipo de escle-

Tabla 5. Diagnóstico anatomopatológico, según edad, de niños con linfoma atendidos en el Instituto Nacional de Salud del Niño de Breña, $2015-2019$. Lima, Perú.

\begin{tabular}{|c|c|c|c|c|}
\hline \multirow[b]{2}{*}{ Diagnóstico anatomopatológico } & \multicolumn{4}{|c|}{ Edad } \\
\hline & Pre escolares & $\begin{array}{c}\text { Porcentaje } \\
\text { (\%) }\end{array}$ & $\begin{array}{c}\text { Escolares } \\
\mathbf{n}\end{array}$ & $\begin{array}{c}\text { Porcentaje } \\
\text { (\%) }\end{array}$ \\
\hline \multicolumn{5}{|l|}{ Linfoma Hodgkin } \\
\hline LH celularidad mixta & 5 & $(31,3)$ & 4 & $(14,8)$ \\
\hline LH esclerosis nodular & 3 & $(18,8)$ & 4 & $(14,8)$ \\
\hline LH rico en linfocitos & 1 & $(6,3)$ & 3 & $(11,1)$ \\
\hline \multicolumn{5}{|l|}{ Linfoma No Hodgkin } \\
\hline Linfoma Linfoblastico B & 4 & $(25,0)$ & 7 & $(25,9)$ \\
\hline Linfoma Linfoblastico T & 1 & $(6,3)$ & 4 & $(14,8)$ \\
\hline Linfoma de Burkitt & 0 & (0) & 3 & $(11,1)$ \\
\hline Linfoma anaplasico de células grandes & 1 & $(6,3)$ & 2 & $(7,4)$ \\
\hline Linfoma difuso de células grandes & 1 & $(6,3)$ & 0 & $(0)$ \\
\hline Total & 16 & $100 \%$ & 27 & $100 \%$ \\
\hline
\end{tabular}


Tabla 6. Diagnóstico anatomopatológico, según sexo, de niños con linfoma atendidos en el Instituto Nacional de Salud del Niño de Breña, 2015 2019. Lima, Perú

\begin{tabular}{|c|c|c|c|c|}
\hline \multirow[b]{2}{*}{ Diagnóstico anatomopatológico } & \multicolumn{4}{|c|}{ Sexo } \\
\hline & $\begin{array}{c}\text { Masculino } \\
\mathbf{n}\end{array}$ & $\begin{array}{c}\text { Porcentaje } \\
\text { (\%) }\end{array}$ & $\begin{array}{c}\text { Femenino } \\
\mathbf{n}\end{array}$ & $\begin{array}{c}\text { Porcentaje } \\
\text { (\%) }\end{array}$ \\
\hline \multicolumn{5}{|l|}{ Linfoma Hodgkin } \\
\hline LH celularidad mixta & 9 & $(29,0)$ & 0 & (0) \\
\hline LH esclerosis nodular & 6 & $(19,4)$ & 1 & $(8,3)$ \\
\hline LH rico en linfocitos & 3 & $(9,7)$ & 1 & $(8,3)$ \\
\hline \multicolumn{5}{|l|}{ Linfoma No Hodgkin } \\
\hline Linfoma linfoblastico B & 8 & $(25,8)$ & 3 & $(25,0)$ \\
\hline Linfoma linfoblastico T & 2 & $(6,5)$ & 3 & $(25,0)$ \\
\hline Linfoma de Burkitt & 2 & $(6,5)$ & 1 & $(8,3)$ \\
\hline Linfoma anaplasico de celulas grandes & 1 & $(3,2)$ & 2 & $(16,7)$ \\
\hline Linfoma difuso de celulas grandes & 0 & $(0)$ & 1 & $(8,3)$ \\
\hline Total & 31 & (100) & 12 & $(100)$ \\
\hline
\end{tabular}

rosis nodular tiene una incidencia mayor en países más ricos ${ }^{(15)}$.

El LH se relaciona con antecedentes de infección por el virus de Epstein-Barr (VEB), ya que las células B en la etapa de diferenciación de células germinales son especialmente propensas a transformación neoplásica. Esto se debe principalmente a que las células $B$ que se someten a hipermutación somática y a recombinación de cambio de clase, son particularmente susceptibles a mutaciones oncogénicas, incluidas las translocaciones cromosómicas, como lo demostró el estudio que realizó Fathima Zumla Cader; en nuestro estudio sólo se halló un caso con antecedente de VEB ${ }^{(16)}$.

En cuanto a la correlación entre linfoma y edad, Worawut y col., reportaron que el linfoma difuso de células B y el linfoma de células grandes fueron evidentes con mayor proporción en adolescentes ${ }^{(5)}$. En nuestro estudio el LNH más frecuente fue el linfocitico $B$, de los 18 casos que se presentaron 11 fueron en pre escolares y 7 en escolares, le sigue en frecuencia el linfoma linfoblástico $T$ con 9 casos siendo 5 en pre escolares y 4 en escolares, siempre a predominio del sexo masculino. Los LNH en los infantes varían con respecto a los adultos en sus características histológicas más limitadas y con alto potencial de malignidad. Se le atribuye alrededor del $60 \%$ de todos los linfomas en la infancia y la adolescencia, representando entre el 8-10\% de las neoplasias en niños entre 5 y 19 años en total ${ }^{(17)}$, destacando el linfoma linfoblástico, causante del $30 \%$ del LNH ${ }^{(18)}$. Por otro lado, debido al elemento linfoide, el anillo de Waldeyer también se ve afectado por la invasión de linfomas, diseminándose por la red linfática y ganglionar a nivel del cuello hacia todo el organismo ${ }^{(19)}$, provocando disnea y disfagia, características clínicas encontradas en nuestro estudio.

Uno de los linfomas que menos casos se reportó fue el linfoma anaplásico de células grandes (LACG), solo hubieron 3 casos; sin embargo, se ha reportado que representan el $10 \%$ de los linfomas de la infancia ${ }^{(20)}$.

Las limitaciones de nuestra investigación son las propias de un estudio retrospectivo. Existieron historias clínicas con datos incompletos, las cuales tuvieron que ser excluidas, asimismo algunas no pudieron ser ubicadas debido a que pertenecían al grupo de pacientes que ya habían cumplido mayoría de edad, siendo dadas de bajas del archivo.

Concluimos que los pacientes con linfoma en el Instituto Nacional de Salud del Niño de Breña tuvieron respecto al sexo, edad y presentación clínica, características similares a las mostradas en la literatura internacional; es decir, mayor frecuencia en varones y en escolares, siendo la fiebre, baja de peso, la presencia de linfoadenopatías, tumoración abdominal y anemia las más frecuentes. El LNH fue más prevalente, siendo el linfoma linfoblástico B el más frecuente.

\section{REFERENCIAS BIBLIOGRÁFICAS}

1. Sánchez de Toledo C, Sábado C. Linfomas de Hodgkin y no Hodgkin. Pediatr Integral. 2016; 20(6): 390-400.

2. Medical News Today [Internet]. MacGill M. Linfoma: Causas, sintomas y tratamientos, 2017 [citado 18 noviembre 2020]. Disponible en: https://www. medicalnewstoday.com/articles/291243\#signos_y _s\%C3\%ADntomas

3. Instituto Nacional de Enfermedades Neoplásicas [Internet]. Payet E, Pérez P, Poquioma E, Dıáz E. Registro de Cáncer de Lima Metropolitana. Incidencia y Mortalidad 2010-2012 [citado 18 noviembre 2020]. Disponible en: http://www.inen.sld. pe/portal/documentos/pdf/banners_2014/2016/Registro\%20de \%20C\%C3\%A1ncer\%20Lima\%20Metropolitana\%202010\%20-\%202012_02092016.pdf

4. Yakubu M, Ahmadu BU, Yerima TS, Simon P, Hezekiah IA, Pwavimbo AJ. Prevalence and clinical manifestation of lymphomas in North Eastern Nigeria. Indian J Cancer. 2015;52:551-5. DOI: 10.4103/0019-509X.178435

5. Tovar JR, Gómez GA. Incidencia de cáncer infantil en una ciudad colombiana. Rev Cienc Salud. 2016;14(03):315-29. DOI: http://dx.doi. org/10.12804/revsalud14.03.2016.01

6. Worawut C, Usanarat A, Nongnuch S, Duantida S, Surapong L, Suradej H, et al. Pediatric non-Hodgkin Iymphoma: Characteristics, stratification, and treatment at a single institute in Thailand. Pediatrics International. 2019; 61(1): 49-57. DOI: 10.1111/ ped.13739

7. Padilla J, Ulloa V, Venegas D. Características epidemiológicas, clínicas y patológicas de los linfomas en el Hospital Nacional Cayetano Heredia del año 1998 al 2008. Acta Méd Per. 2011:28(1):12-8. 
8. Del Castillo R. Clinical, epidemiological and treatment response characteristics of patients with Non Hodgkin Lymphoma at National Dos de Mayo Hospital: 2004-2011, Lima-Peru. Revista Médica Carrionica. 2017;4(1):1-21.

9. Chang A, Seminario V, Navarrete J, Melendez R, Torres E, Barrionuevo C, et al. Linfomas extraganglionares en Lima Metropolitana. An Fac med. 2001;62(3): 193-199. DOI: https://doi.org/10.15381/ anales.v62i3.4173

10. Bencomo B, Herrera L. Caracterización clínico epidemiológico de los linfomas en laedad pediátrica en Pinar del Río, 2010-2016. Rev Ciencias Médicas. 2017;21(4): 41-47.

11. Acerca del linfoma no Hodgkin [Internet]. American Cancer Society, 2018 [citado 10 enero 2021]. Disponible en: https://www.cancer.org/content/dam/ CRC/PDF/Public/9079.00.pdf.
12. Vela E, Cruz O. Linfomas. En: Cruz M. Tratado de Pediatria. t-2. España: Editorial Médica Panamericana; 2013.

13. Mingo E, Chacón J, Padilla M. Fisiopatología del anillo Waldeyer. $1^{\text {a }}$ Ed. Madrid: Sociedad española de Otominolaringologíay Patología Cérvico-Facial SEORL-PCF;2014

14. Instituto Nacional del Cáncer [Internet]. Tratamiento del linfoma de Hodgkin infantil $(P D Q \otimes)$ - Versión para pacientes, 2020 [citado 9 de diciembre de 2020]. Disponible en: https://www.cancer.gov/espanol/tipos/ linfoma/paciente/tratamiento-hodgkin-infantil-pdq

15. AEAL [Internet]. Linfoma de Hodgkin. España: Asociación Española de Afectados por Linfoma, Mieloma y Leucemia [citado el 20 de febrero del 2017]. Disponible desde: http://www.aeal.es/linfoma-de-hodgkin-espana/2-epidemiologia/\#2-1LH

16. Cader F, Kearns P, Young L, Murray P, Vockerod $M$. The contribution of the Epstein-Barr virus to the pathogenesis of childhood lymphomas.
Cancer Treatment Reviews. 2010;36:348-53. DOI: 10.1016/j.ctrv.2010.02.011

17. Kliegman RM, Behrman RE, Jenson HB, Stanton BF. Nelson Tratado de Pediatria,18va Ed., España. 2009.

18. Bonn BR, Rohde M, Zimmermann M, Krieger D, Oschlies I, Niggli F, et al. Incidence and prognostic relevance of genetic variations in T-cell lymphoblastic lymphoma in childhood and adolescence. Blood. 2013;121(16): 3153-3160. DOI: 10.1182/ blood-2012-12-474148

19. Mingo E, Chacón J, Padilla M. Fisiopatologia del anillo Waldeyer. $1^{\text {a }}$ Ed. Madrid: Sociedad española de Otorrinolaringología y Patología Cérvico-Facial SEORL-PCF; 2014

20. Escobosa O, Herrero A, Acha T. Linfoma anaplásico de células grandes Endo bronquial en la infancia. An Pediatr (Barc). 2009;70(5):449-452. DOI: 10.1016/j.anpedi.2008.12.008 